\title{
TURBO EQUALIZATION OF CONVOLUTIONAL CODED AND CONCATENATED SPACE TIME TRELLIS CODED SYSTEMS USING RADIAL BASIS FUNCTION AIDED EQUALIZERS
}

\author{
M. S. Yee, B. L. Yeap and L. Hanzo \\ Dept. of Electr. and Comp. Sc., Univ. of Southampton, SO17 1BJ, UK. \\ Tel: +44-(0)23-8059 3125, Fax: +44-(0)23-8059 3045 \\ Email: 1h@ecs.soton.ac.uk \\ http://www-mobile.ecs.soton.ac.uk
}

\begin{abstract}
In this contribution a reducedcomplexity radial basis function (RBF) aided neural-network based turbo equalization (TEQ) scheme is proposed for employment in a serially concatenated convolutional coded and systematic space time trellis coded (CC-SSTTC) arrangement. A two-path Rayleigh fading channel having a normalised Doppler frequency of $3.3615 \times 10^{-5}$ was used. The BER performance of the RBF-CC-SSTTC $(4,4)$ scheme employing a transmission burst consisting of 100 symbols using a space-time-trellis (STT) interleaver of at least 400 symbols and eight turbo equalization iterations was found to be similar to that of the CC-SSTTC system using a trellis-based TEQ, which attains the optimum performance. However, the Jacobian RBF based TEQ provided a complexity reduction factor of 14 .
\end{abstract}

\section{INTRODUCTION}

The third-generation proposals aim for guaranteeing low-cost, high-capacity mobile communications offering data rates of up to $2 \mathrm{Mbps}$ [1]. However, there are a number of problems associated with high data rate transmissions, especially when aiming for creating spectrally efficient systems. Systems transmitting at high bit rates, such as $2 \mathrm{Mbps}$, experience a high grade of channel-induced dispersion and suffer from Inter Symbol Interference (ISI). Therefore, typically channel equalizers are employed for mitigating the effects of ISI. In addition to channel equalization, channel coding can also be invoked for further improving the performance of the system. Powerful error correction schemes, such as turbo codes [2], have been shown to yield a performance close to Shannonian performance limits. The discovery of turbo codes and turbo decoding led to the development of turbo equalization [3]. Turbo equalization is a technique that performs chan- nel equalization and channel decoding jointly and iteratively. This scheme has been shown to successfully mitigate the effects of channel-induced ISI, resulting in a Bit Error Rate (BER) performance close to that recorded for transmission over non-dispersive Gaussian channels.

The family of transmission diversity techniques referred to as Space Time Trellis (STT) coding [4] provides a substantial diversity gain for mobile stations by upgrading the base stations, hence potentiallly increasing the achievable user capacity of the system. STT coding [4] jointly designs the channel coding, modulation, transmit diversity and the optional receiver diversity schemes invoked. Following the research by Tarokh et al. [4], Bauch et al. [5] proposed a joint equalization and STT decoding scheme, which yielded an improved performance with the advent of exploiting the soft-decision based feedback from the STT decoder's output to the channel equalizer's input. In [6] the performance of the STT encoded system was further improved by employing additional channel encoding in conjunction with turbo equalization. We refer to this turbo equalizer as the TEQ-STTC scheme. However, due to the associated computational complexity, the employment of this scheme was limited to low-order modulation modes, such as for example 4-level Quadrature Amplitude Modulation (4QAM).

Motivated by these trends, in this contribution we aim for reducing the complexity associated with the channel-coded and concatenated STT encoded system by using a reduced-complexity Jacobian Radial Basis Function (RBF) equalizer [7], which we will refer to as the RBF-TEQ-STTC scheme. We will investigate the BER performance achieved by the RBF-TEQSTTC scheme and evaluate the achievable computational complexity reduction compared to the conventional trellis-based TEQ-STTC (CT-TEQ-STTC) arrangement of $[6]$. 


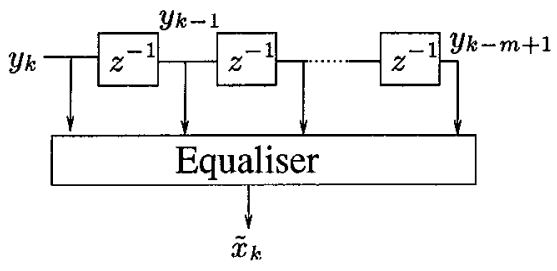

Figure 1: Schematic of $m$-tap equalizer

\section{RBF AIDED CHANNEL EQUALIZER FOR SPACE-TIME-CODING}

In this section we will show that the channel equalization problem encountered in a space-time coded system can be considered as a geometric classification problem [8], namely that of classifying an $\mathcal{M}$-ary received phasor into one of $\mathcal{M}$ classes. Figure 1 shows an $m$-tap equalizer schematic, where the channel output observed by the equalizer can be written in vectorial form as

$$
\mathbf{y}_{k}=\left[\begin{array}{llll}
y_{k} & y_{k-1} & \ldots & y_{k-m+1}
\end{array}\right] .
$$

The baseband representation of the $p$-transmitter spacetime coded system is shown in Figure 2, which transmits a sequence of $p$ symbols $\mathbf{x}_{k}^{p}=\left[\begin{array}{lll}x_{1, k} & \ldots & x_{p, k}\end{array}\right]$ during each signalling instant $k$. The channel output at instant $k$ is given by:

$$
y_{k}=\sum_{i=1}^{p} \mathbf{h}_{i} \mathbf{x}_{i, k}^{T}+\eta_{k},
$$

where the $i$-th channel impulse response (CIR) $\mathbf{h}_{i}=$ $\left[\begin{array}{llll}h_{0, i} & h_{1, i} & \ldots & h_{L}\end{array}\right]$, having a memory of $L$ symbols, is convolved with a sequence of $L+1$ transmitted symbols, namely with $\mathbf{x}_{i, k}=\left[\begin{array}{lll}x_{i, k} & x_{i, k-1} & \ldots\end{array}\right.$ $\left.x_{i, k-L}\right]$ and $\eta_{k}$ is the additive Gaussian noise term having a variance of $\sigma_{\eta}$. For a $p$-transmitter system using an $m$-tap equalizer and communicating over a channel having a CIR memory of $L$ (assumming that all of the $p$ CIRs have the same memory), there are $n_{s}=$ $\mathcal{M}^{(m+L) \cdot p}$ number of possible received phasor combinations due to the transmitted sequence, hence producing $n_{s}$ number of different possible channel output vectors in the absence of channel noise:

$$
\tilde{\mathbf{y}}_{k}=\left[\begin{array}{llll}
\tilde{y}_{k} & \tilde{y}_{k-1} & \ldots & \tilde{y}_{k-m+1}
\end{array}\right],
$$

where $m$ is the length of the equalizer in Figure 1. Upon adding the noise we have: $\mathbf{y}_{k}=\tilde{\mathbf{y}}_{k}+\eta_{k}$. Expounding further, we denote each of the $n_{s}$ number of different possible combinations of the channel's input sequence $\overline{\mathbf{x}}_{k}=\left[\begin{array}{lll}\mathbf{x}_{k}^{p} & \ldots & \mathbf{x}_{k-m+1}^{p}\end{array}\right]$ of length $(L+m) \times p$ symbols as $\mathbf{s}_{i}, i=1, \ldots, n_{s}$, where the channel's input state $\mathbf{s}_{i}$ determines the desired channel output state $\mathrm{r}_{i}, i=1, \ldots, n_{s}$. This is formulated as:

$$
\tilde{\mathbf{y}}_{\mathbf{k}}=\mathbf{r}_{i}, \quad \text { if } \overline{\mathbf{x}}_{k}=\mathbf{s}_{i}, \quad i=1, \ldots, n_{s} .
$$

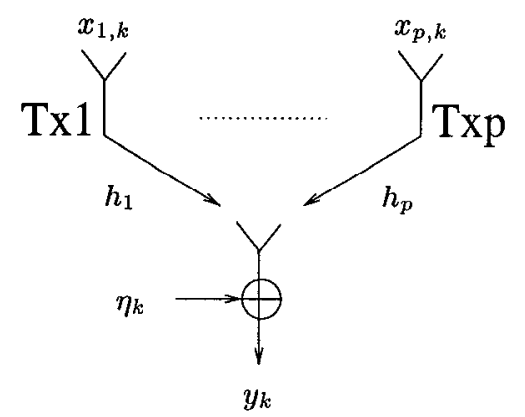

Figure 2: Baseband representation of $p$-transmitter space-time coded system using one receiver.

For an $\mathcal{M}$-level modulation scheme, the noisy channel output states $\mathbf{y}_{k}$ can be partitioned into $\mathcal{M}^{p}$ classes according to the sequence of $p$ number of $\tau$-delayed transmitted symbols, $x_{k-\tau}^{p}$. The equalizer has to provide the associated non-linear decision boundaries for the classification strategy. The optimum equalizer is the so-called Bayesian equalizer [8], which has an excessive complexity. Hence here we advocate the reducedcomplexity, but suboptimum Jacobian RBF equalizer, introduced in [7], which has $N$ hidden nodes. The output of this Jacobian RBF equalizer can be represented mathematically as [7]:

$$
\begin{aligned}
f_{R B F}^{\ln }\left(\mathbf{y}_{k}\right) & =\ln \left(\sum_{i=1}^{N} w_{i} \exp \left(-\left\|\mathbf{y}_{k}-\mathbf{c}_{i}\right\|^{2} / \lambda\right)\right) \\
& =\ln \left(\sum_{i=1}^{N} \exp \left(\ln \left(w_{i}\right)-\left\|\mathbf{y}_{k}-\mathbf{c}_{i}\right\|^{2} / \lambda\right)\right) \\
& =J\left(\delta_{N, k}, J\left(\delta_{N-1, k}, \ldots J\left(\delta_{2, k}, \delta_{1, k}\right) \ldots\right)\right),
\end{aligned}
$$

where the terms $w_{i}, \mathbf{c}_{i}$ and $\lambda$ are the weights, centers and width of the RBF nodes, respectively. Furthermore, we have $\delta_{i, k}=\exp \left(\ln \left(w_{i}\right)-\left\|\mathbf{y}_{k}-\mathbf{c}_{i}\right\|^{2} / \lambda\right)$ and $J\left(\delta_{1}, \delta_{2}\right)$ is the Jacobian logarithmic relationship defined in [9] as $J\left(\delta_{1}, \delta_{2}\right) \approx \max \left(\delta_{1}, \delta_{2}\right)+f_{c}\left(\left\|\delta_{1}-\delta_{2}\right\|\right)$. The correction function $f_{c}(x)=\ln (1+\exp (-x))$ is tabulated in a look-up table, in order to reduce the computational complexity [9].

The full-complexity RBF equalizer provides the socalled optimal Bayesian equalization solution [8] and generates the conditional probability density functions of $\mathcal{M}^{p}$ number of possible transmitted symbols $\mathrm{x}_{k-\tau}^{p}$ emitted by the transmitters at instant $k-\tau$ in the form of:

$$
\begin{aligned}
& P\left(\mathbf{y}_{k} \mid \mathbf{x}_{k-\tau}^{p}=\mathbf{I}_{j}\right)= \sum_{i=1}^{n_{s}^{j}} p_{i, j}\left(2 \pi \sigma_{\eta}^{2}\right)^{-m / 2} . \\
& \exp \left\{-\frac{1}{2 \sigma_{\eta}^{2}}\left\|\mathbf{y}_{k}-r_{i, j}\right\|^{2}\right\}, \\
& j=1, \ldots, \mathcal{M}^{p}
\end{aligned}
$$


where the RBF parameters defined in the context of Equation 6 are assigned the values of $\mathrm{w}_{i}=p_{i, j}\left(2 \pi \sigma_{\eta}^{2}\right)^{-m / 2}$, $\mathbf{c}_{i}=r_{i, j}, N=n_{s}^{j}$ and $\lambda=2 \sigma_{\eta}^{2}$. The term $n_{s}^{j}$ is the number of possible channel states $\mathbf{r}_{i, j}$ corresponding to the $j$ th transmitted symbol sequence $\mathbf{I}_{j}$ of the $p$-antenna SSTTC scheme that consist of $p$ symbols, where we have $j=1, \ldots, \mathcal{M}^{p}$. The term $p_{i, j}$ is the a priori probability of occurance of the channel state $r_{i, j}$. The a posteriori probability of the transmitted symbols $\mathrm{x}_{k-\tau}^{p}$ in Equation 6 provides the a posteriori Log-Likelihood Ratio (LLR) values of the convolutionally coded symbols, which can then be fed to the STT decoder, as shown in Figure 4. The a priori probability of occurance of the $i$ th channel state $\mathbf{r}_{i, j}$ corresponding to the transmitted symbol sequence $\mathbf{I}_{j}, p_{i, j}$, can be evaluated from the LLRs generated by the STT decoder as described in Section 3.

\section{SYSTEM OVERVIEW}

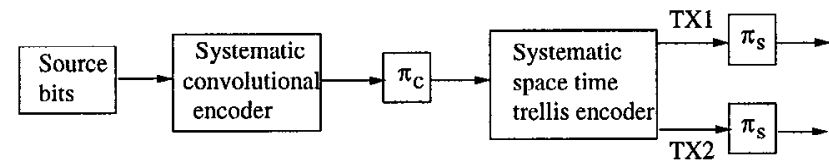

Figure 3: Transmitter of the serially concatenated systematic convolutional coded and systematic STT coded system.

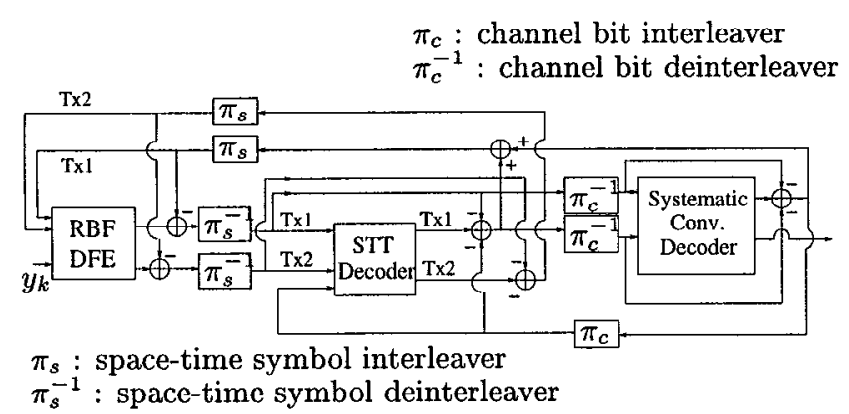

Figure 4: Receiver of the serially concatenated systernatic convolutional coded and systematic STTC system using RBF DFE assisted turbo equalization.

In an effort to create a low-complexity, high-performance system, we employ the Jacobian RBF equalizer [7] in the context of a turbo equalizer in conjunction with a convolutional coded systematic STTC (CCSSTTC) system employing two transmitters. Specifically, we use the decision feedback assisted Jacobian RBF equalizer (Jacobian RBF DFE) [7] for the sake of attaining a reduced computational complexity, where the detected symbol is fed back to the equalizer for selecting a reduced-size subset of RBF centers, which are then used for evaluating the a posteriori probability of the transmitted signal [8]. The schematic of the CC-SSTTC transmitter consists of a serially concatenated systematic convolutional encoder and a systematic STT encoder, as shown in Figure 3. The transmitted source bits are convolutionally encoded and directed to a random channel bit interleaver $\pi_{c}$. The convolutional encoder denoted as $\mathrm{CC}(2,1,3)$ is a $\frac{1}{2}$-rate Recursive Systematic Convolutional (RSC) coding scheme having a constraint length of $K=3$ and octal generator polynomials of $G_{0}=7$ and $G_{1}=5$. The RSC codeword consists of a systematic bit and a parity bit.

Subsequently, the encoded bits are passed to a systematic STT encoder using two transmit antennas, as illustrated in Figure 3. We denote the systematic STT encoder used as the $\operatorname{SSTTC}(n=4, m=4)$ scheme, since it is an $n=4$-state, $m=4$-PSK based STT code [4]. Upon receiving an input symbol, the SSTTC produces a symbol in each transmitter arm of Figure 3. Note that we have employed the simple $\operatorname{SSTTC}(4,4)$ code instead of more complex systematic STT codes using a highcr number of cncoder statcs, sincc our aim was to invoke the turbo equalization principle and 'invest' the affordable implementational complexity in a number of consecutive iterations, rather than in a highcomplexity non-iterative decoder. The STT encoded symbols are interleaved by a random STT symbol interleaver represented as $\pi_{s}$ in Figure 3.

The schematic of the receiver is shown in Figure 4. The channel equalizer of Figure 4 computes the a posteriori LLR values for the systematic STT coded symbols of both transmitter TX1 and TX2. Subsequently, these LLR values are deinterleaved by the STT deinterleaver $\pi_{s}^{-1}$ of Figure 4 and passed to the $\operatorname{SSTTC}(4,4)$ decoder. In the first iteration, the channel equalizer only evaluates the received signal $y_{k}$, since there is no a priori feedback information from the outpul of the RSC decoder. However, in subsequent iterations the channel equalizer will receive additional a priori information concerning the STT codeword from the other decoding stages. In order to avoid passing the a priori information contributed by the other concatenated decoding states back to these stages in Figure 4, we subtract the a priori LLRs fed back to the input of the equalizer from the corresponding a posteriori LLRs output by the equalizer, in order to derive the combined channel and extrinsic information. Similar LLR subtraction stages can be seen at the output of the STT decoder and that of the convolutional decoder, again providing the extrinsic information for the next component of the receiver, as detailed in [6].

In our investigations the transmission burst structure consists of 100 data symbols. A two-path, symbolspaced fading Channel Impulse Response (CIR) of equal weights was used, where the Rayleigh fading statis- 
tics obeyed a normalised Doppler frequency of $3.3615 \times$ $10^{-5}$. The fading magnitude and phase was kept constant for the duration of a cransmission burst, a condition which we refer to as employing burst-invariant fading. Furthermore, in order to investigate the bestcase performance of these systems, we have assumed that the CIR was perfectly estimated at the receiver. Our future research will characterise the ability of the proposed turbo scheme to compensate for the effects of CIR estimation errors. At the receiver, the systematic STT decoder and the RSC decoder employed the Log-MAP algorithm [9]. The Jacobian RBF DFE has a feedforward order of $m=2$, feedback order of $n=1$ and decision delay of $\tau=1$.

\section{RESULTS AND DISCUSSION}

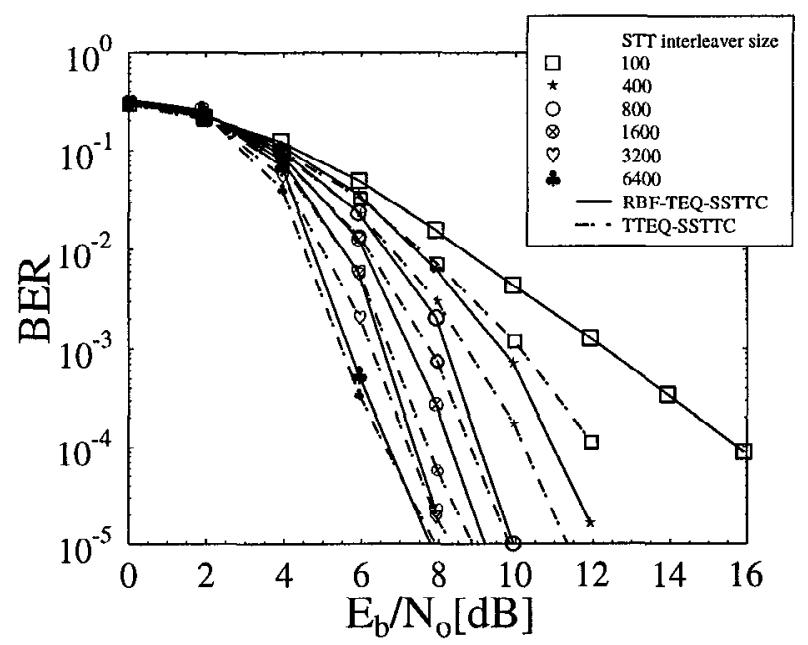

Figure 5: BER performance of the RBF DFE ( $m=2$, $n=1, \tau=1$ ) assisted turbo-equalized serially concatenated convolutional coded and STTC system using various STTC interleaver sizes, namely 100, 400, $800,1600,3200$ and 6400 symbols, after eight turbo equalization iterations. The performance of the CTTEQ-SSTTC system is also shown as a benchmarker.

Figure 5 shows the performance of the proposed RBF-TEQ-STTC and that of the CT-TEQ-STTC scheme [6], using various STTC interlcaving sizcs, namcly $100,400,800,1600,3200$ and 6400 symbols after eight turbo equalization iterations. It was observed in Figure 5 that by increasing the STTC interleaving size from 100 to 6400 , the performance degradation of the RBF-TEQ-STTC scheme compared to the CT-TEQSTTC arrangement expressed in terms of the excess SNR required for attaining a $\mathrm{BER}$ of $10^{-4}$ decreases from $3.8 \mathrm{~dB}$ recorded for an STTC interleaver size of 100 symbols to $0 \mathrm{~dB}$, as observed for the STTC interleaver size of 6400 symbols. This is because the error propagation of the RBF DFE component decreases, as the BER performance improves, when using a longer STTC interleaver. The performance difference of the two schemes is less than $1 \mathrm{~dB}$ at a STTC interleaver length of 400 symbols, although the RBF-TEQ-STTC scheme has a lower computational complexity, when the feedforward order $m$ and feedback order $n$ are set to $m=L+1, n=L$. The interleaving gain attained by the RBF-TEQ-STTC scheme was approximately $9 \mathrm{~dB}$ at a BER of $10^{-4}$. Although higher interleaving gains can be achieved using longer STTC interleavers, the interleaver gain gradually saturates, when the STTC interleaver size is in excess of 1600 symbols.

\begin{tabular}{|lll|}
\hline & trellis-based & Jacobian RBF \\
\hline sub. and add. & $n_{s, f}\left(6 \mathcal{M}^{p}+2\right)$ & $n_{s, f}+$ \\
& -3 & $\mathcal{M}^{p} n_{s}^{i}(m+2)-4$ \\
mult. and div. & $2 n_{s, f}$ & $2 n_{s, f}$ \\
\hline
\end{tabular}

Table 1: Computational complexity of gencrating the a posteriori LLRs for the trellis-based equalizer and for the Jacobian RBF equalizer [10]. The RBF equalizer's feedforward and feedback order are denoted by $m$ and $n$, respectively, and the number of RBF nodes is $n_{s}^{i}=\mathcal{M}^{(m+L-n) \cdot p} / \mathcal{M}, i=1, \ldots, \mathcal{M}^{p}$, where $L$ is the CIR memory and $p$ is the number of STTC transmitters. The notation $n_{s, f}=\mathcal{M}^{(L+1) \cdot p}$ indicates the number of trellis transitions encountered in the trellisbased equalizer and also the number of possible different noise-free channel outputs $\tilde{y}_{k}$ of the Jacobian RBF equalizer.

Following the approach of our computational complexity study in [10], Table 1 summarises the computational complexity of generating the a posteriori LLRs for each received signal at instant $k$ in the context of a $p$-transmitter space-time coded system. Figure 6 demonstrates the complexity reduction achieved by the Jacobian RBF DFE for various feedforward orders $m$, over the trellis-based equalizer. The feedback order $n$ and decision delay $\tau$ of the RBF DFE was set to $n=L$ and $\tau=m$ for the sake of attaining the optimum performance, as stated in [8]. The performance of the RBF DFE improves, when increasing the feedforward order [8]. Howcver, Figurc 6 shows that the Jacobian RBF DFE only provides a significant complexity reduction compared to the trellis based equalizer, when the feedforward order is less than $L+2$ and imposes a higher computational complexity for $m>L+2$. Therefore, as a rule of thumb, the feedforward order of the Jacobian RBF DFE must not exceed $L+1$ in order to achieve a computational complexity improvement relative to the trellis-based equalizer. The complexity imposed by the RBF-TEQ-STTC scheme using an equalizer feedforward order of 2 and a feedback order of 1 was found 


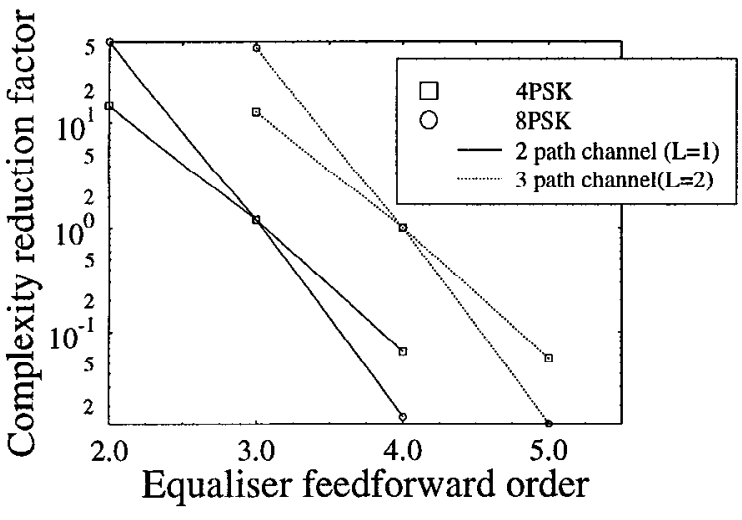

Figure 6: Complexity reduction factor achieved by the RBF DFE equalizer over the trellis based equalizer according to Table 1 . The feedback order $n$ was set to $L$ and the number of transmitters was two.

to be a factor of 14 lower, than that of the CT-TEQSTTC scheme in the context of a two transmitter, one receiver system, based on the general complexity expressions of Table 1. For example, if we used a higher order modulation mode, such as 8PSK used in [4] along with the same number of transmitters, as well as equalizer and channel parameters, the achievable computational complexity reduction is a factor of 55 , as shown in Figure 6.

\section{CONCLUSIONS}

A turbo equalization scheme using the Jacobian RBF equalizer principle of [7] was invoked in a serially concatcnated systematic convolutional coded and systematic STT coded system. It was observed in Figure 5 that the BER performance degradation compared to the CT-TEQ-STTC system [6] was less than $1 \mathrm{~dB}$ for a STTC interleaver length of 400 symbols, while achieving a computational complexity reduction factor 14 . Hence, the Jacobian RBF equalizer based TEQ constitutes a better design choice in STTC systems, especially in the context of complex STTC schemes, having a high number of encoder states. Near-optimum performance was achieved, provided that a sufficiently high STTC interleaver length was affordable.

\section{REFERENCES}

[1] T. Ojanperä and R. Prasad, Wideband CDMA for Third Generation Mobile Communications. Artech House, 1998.

[2] C. Berrou, A. Glavieux, and P. Thitimajshima, "Near Shannon Limit Error-Correcting Coding and Decoding: Turbo Codes," in Proceedings of the International Conference on Communications, (Geneva, Switzerland), pp. 1064-1070, 23-26 May 1993.

[3] C. Douillard, A. Picart, M. Jézéquel, P. Didier, C. Berrou, and A. Glavieux, "Iterative correction of intersymbol interference: Turboequalization," European Transactions on Communications, vol. 6, pp. 507-511, September-October 1995.

[4] V. Tarokh, N. Seshadri, and A. R. Calderbank, "Space-Time Codes for High Data Rate Wireless Communication: Performance Criterion and Code Construction," IEEE Transactions on Information Theory, vol. 44, pp. 744-765, March 1998.

[5] G. Bauch, A. F. Naguib, and N. Seshadri, "MAP Equalization of Space-time Coded Signals over Frequency Selective Channels," in Proceedings of the Wireless Communications and Networking Confeerence, (New Orleans, USA), pp. 261-265, September 1999.

[6] B. L. Yeap, T. H. Liew, and L. Hanzo, "Turbo equalization of serially concatenated systematic convolutional codes and systematic space time trellis codes," in Proceedings of the IEEE Vehicular Technology Conference, (Rhodes, Greece), 6 9 May 2001. CD-ROM.

[7] M. S. Yee, T. H. Liew, and L. Hanzo, "Block turbo coded burst-by-burst adaptive radial basis function decision feedback equaliser assisted modems," in Proceedings of IEEE Vehicular Technology Conference, vol. 3, (Amsterdam, Netherlands), pp. 1600-1604, September 1999.

[8] S. Chen, S. McLaughlin, and B. Mulgrew, "Complex-valued radial basis function network, Part II: Application to digital communications channel equalisation," EURASIP Signal Processing, vol. 36, pp. 175-188, March 1994.

[9] P. Robertson, E. Villebrun, and P. Hoeher, "A Comparison of Optimal and Sub-Optimal MAP Decoding Algorithms Operating in the Log Domain," in Proceedings of the International Conference on Communications, (Seattle, United States), pp. 1009-1013, 18-22 June 1995.

[10] M. S. Yee, B. L. Yeap, and L. Hanzo, "Radial Basis Function Assisted Turbo Equalisation," in Proceedings of the IEEE Vehicular Technology Conference 2000 , pp. 640-644, 2000. 